\title{
Estenosis de la arteria mesentérica superior como causa de isquemia crónica intestinal. Tratamiento percutáneo como alternativa terapéutica
}

\author{
$\mathrm{M}^{\mathrm{a}}$ D. FERRER, J. GIL, J. GUIJARRO, I. PASCUAL*, E. PARREÑO \\ Servicios de Radiodiagnóstico y *Gastroenterología. Hospital Clínico Universitario. \\ Valencia
}

\begin{abstract}
CHORNIC MESENTERIC ISCHEMIA DUE TO SUPERIOR MESENTE RIC ARTERY STENOSIS. PERCUTANEOUS THERAPY AS ALTERNA TIVE TREATMENT
\end{abstract}

\section{RESUMEN}

La isquemia intestinal crónica es un cuadro poco frecuente que se encuentra asociado a una alta morbilidad y mortalidad. La causa más frecuente es la arteriosclerosis. Los pacientes sufren dolor abdominal localizado en epigastrio o periumbilical que aparece de 10 a 30 minutos después de la ingesta.

Presentamos un caso de isquemia intestinal crónica por estenosis de la arteria mesentérica superior diagnosticado mediante ecografía doppler y angiografía, que se trató con angioplastia percutánea con resolución completa de la clínica. Se comentan los hallazgos clínicos-radiológicos y se describe el procedimiento terapéutico.

Pensamos que el tratamiento percutáneo ofrece un beneficio en este tipo de patología, disminuyendo el número de complicaciones, reduciendo la estancia hospitalaria y aumentando el grado de confort para los pacientes.

PALABRAS CLAVE: Arteria mesentérica. Isquemia mesentérica. Angioplastia percutánea transluminal.

\section{ABSTRACT}

Chronic mesenteric arterial ischemia is an uncommon condition associated with a high morbidity and mortality. It is most commonly cau sed by atherosclerotic occlusive disease. Patients may suffer epigastric or periumbilical post-prandial pain ten to thirty minutes after eating. A case of chronic mesenteric ischemia is presented due to superior mesen teric artery stenosis. The diagnosis was performed with doppler sonno graphy and angiography and was treated with percutaneous translumi nal angioplasty. The patient became completely asymptomatic. The purpose of this report is to present the case, clinic and radiological fea tures and to describe the percutaneous procedure.

We believe that percutaneous treatment offers an improvement in this pathology with a low complication rate, decreasing the admission days and increasing patient confort degree.

KEY WORDS: Arteries mesenteric. Mesentery ischemia. Percutaneous transluminal angioplasty.

Ferrer $M^{a} D$, Gil J, Guijarro J, Pascual I, Parreño E. Estenosis de la arteria mesentérica superior como causa de isquemia crónica intestinal. Tratamiento percutáneo como alternativa terapéutica. An Med Interna (Madrid) 2002; 19: 576-578.

\section{INTRODUCCIÓN}

La isquemia intestinal es un cuadro poco frecuente que está asociado a una alta morbilidad y mortalidad. La rareza de esta entidad se debe a la gran cantidad de colaterales vicariantes que existen en los vasos viscerales.

El síntoma principal de la isquemia mesentérica crónica (IMC) es el dolor abdominal post-prandial, localizado en el epigástrico o periumbilical, que aparece típicamente de 10 a 30 minutos después de la ingesta y presenta una duración aproximada de 1 a 3 horas. El dolor aumenta progresivamente en frecuencia y severidad, por lo que el paciente reduce la ingesta $o$ evita comer, con la consiguiente pérdida de peso. En ocasiones, aparecen nauseas, vómitos y diarreas que aunque no son específicas de esta enfermedad pueden estar presentes (1-3).

Aunque los estudios angiográficos muestran con frecuencia oclusiones parciales o completas del tronco celiaco, de la arteria mesentérica superior y de la arteria mesentérica infe- rior, la IMC es rara. El diagnóstico está basado en las manifestaciones clínicas, la demostración angiográfica de oclusiones arteriales esplácnicas, la exclusión de otra enfermedad que justifique el cuadro clínico, y la respuesta clínica a la revascularización (4). En cuanto al tratamiento, en los últimos 10 años se han introducido varias innovaciones en el manejo terapéutico de esta entidad (2,3).

Presentamos un caso de isquemia intestinal por estenosis de la arteria mesentérica superior diagnosticado mediante ecografía doppler y angiografía, que se trató con angioplastia percutánea con resolución completa. Se comentan los hallazgos clínicosradiológicos y se describe el procedimiento terapéutico.

\section{CASO APORTADO}

Paciente de 72 años, sin antecedentes médico-quirúrgicos de interés, que ingresa en el Servicio de Gastroenterología por presentar en los últimos 2 meses dolor abdominal en epi-mesogastrio irradiado al resto

Trabajo aceptado: 28 de diciembre de 1999

Correspondencia: M $^{\mathrm{a}}$ Dolores Ferrer Puchol. Servicio de Radiodiagnóstico. Sección de Rx Vascular. Hospital Clínico Universitario. Avda, Blasco Ibáñez, 17. 46010 Valencia. 
del abdomen que aparece tras la ingesta, y se acompaña ocasionalmente de vómitos. La paciente refería que había adelgazado $6 \mathrm{Kg}$ desde el inicio del cuadro, lo cual atribuía a la pérdida del apetito y a que tenía verdadero miedo a comer porque le provocaba dolor. No presentaba alteración del ritmo defecatorio. No tomaba anti-inflamatorios.

A la exploración física al ingreso, la paciente presentaba una TA 130/60 mm Hg, con una frecuencia cardiaca de 60 ppm y estaba apirética. Tenía buen aspecto general, con buena coloración e hidratación de piel y de mucosas. El abdomen era blando, depresible, no doloroso a la palpación, sin detectarse masas ni visceromegalias, los ruidos intestinales eran normales y no se auscultó ningún soplo abdominal. La auscultación cardíaca y pulmonar era normal.

En cuanto a las exploraciones complementarias efectuadas, objetivamos que el hemograma, coagulación y la bioquímica hemática incluyendo marcadores tumorales (CEA y CA 19,9) presentaban valores dentro de la normalidad.

Se realizó una radiografía simple de abdomen que no mostraba alteraciones, la ecografía abdominal presentaba litiasis biliar como único hallazgo, siendo el resto del abdomen normal. El TC abdominal demostraba un páncreas atrófico, no objetivándose hallazgos patológicos en el resto del abdomen y pelvis. Se realizó una gastroscopia que demostró lesiones agudas de la mucosa gástrica, con una duodenitis erosiva; se tomaron biopsias del antro gástrico y el estudio histológico mostraba una gastritis crónica atrófica con gérmenes de helicobácter pylori.

Ante la sospecha de patología vascular se decidió realizar una ecografía doppler, que puso de manifiesto la presencia de una estenosis marcada en la AMS aproximadamente a $1 \mathrm{~cm}$ del ostium de salida; el análisis del espectro de flujo obtenido en la zona de mayor velocidad mostró una velocidad telediastólica marcadamente elevada (312 $\mathrm{cm} / \mathrm{seg}$ ), lo que indicaría una estenosis del $90 \%$ en el lugar indicado.

Ante estos hallazgos se decidió la realización de una arteriografía abdominal, demostrando en la aortografía la nula representación de la arteria mesentérica superior, por lo que se realizó una arteriografía selectiva utilizando un catéter visceral evidenciándose una estenosis concéntrica y crítica (más del $90 \%$ de su calibre) de la arteria mesentérica superior localizada a $1 \mathrm{~cm}$ del ostium de salida (Fig. 1), dato que concordaba con los hallazgos ecográficos. Con ayuda de una guía hidrofílica se franqueó la estenosis y se realizó el intercambio con una guía rígida sobre la que se introdujo un catéter de balón de angioplastia de $4 \mathrm{~mm}$ de diámetro que se hinchó utilizando un manómetro hasta conseguir la total dilatación del vaso. Durante la manipulación arterial se administraron 5.000 U intra-arteriales de heparina y 200 microgramos intra-arteriales de nitroglicerina para prevenir el espasmo arterial. El resultado morfológico fue bueno y la paciente fue dada de alta dos días después del procedimiento con tratamiento antiagregante plaquetario, con buena tolerancia a la ingesta y con la desaparición de las molestias abdominales en su totalidad.

La paciente permaneció asintomática durante dos meses pero a partir de los mismos comenzó con dolores abdominales tras la ingesta que, habían variado en la localización y en el momento de su aparición; ahora eran en meso e hipogastrio y aunque tenían relación con las comidas aparecían más tardíamente.

En la exploración destacaba pérdida de $5 \mathrm{~kg}$ de peso en los 20 días previos ya que la enferma prácticamente se negaba a comer. Los datos analíticos y de exploración física eran similares a los encontrados en el primer episodio.

Se realizó nueva arteriografía selectiva de la arteria mesentérica superior que puso de manifiesto una representación normal de las ramas yeyunales con una disminución importante de calibre de la arteria a partir de éstas observándose un segmento estenótico localizado más distalmente que en el episodio anterior. La porción angioplastiada se mantenía de calibre normal. Nuevamente se procedió a la angioplastia con un balón de dilatación de $3 \mathrm{~mm}$ obteniendo un resultado morfológico bueno con representación de todas las ramas distales de la arteria (Fig 2).

La paciente evolucionó favorablemente, estando en la actualidad asintomática 4 meses después.

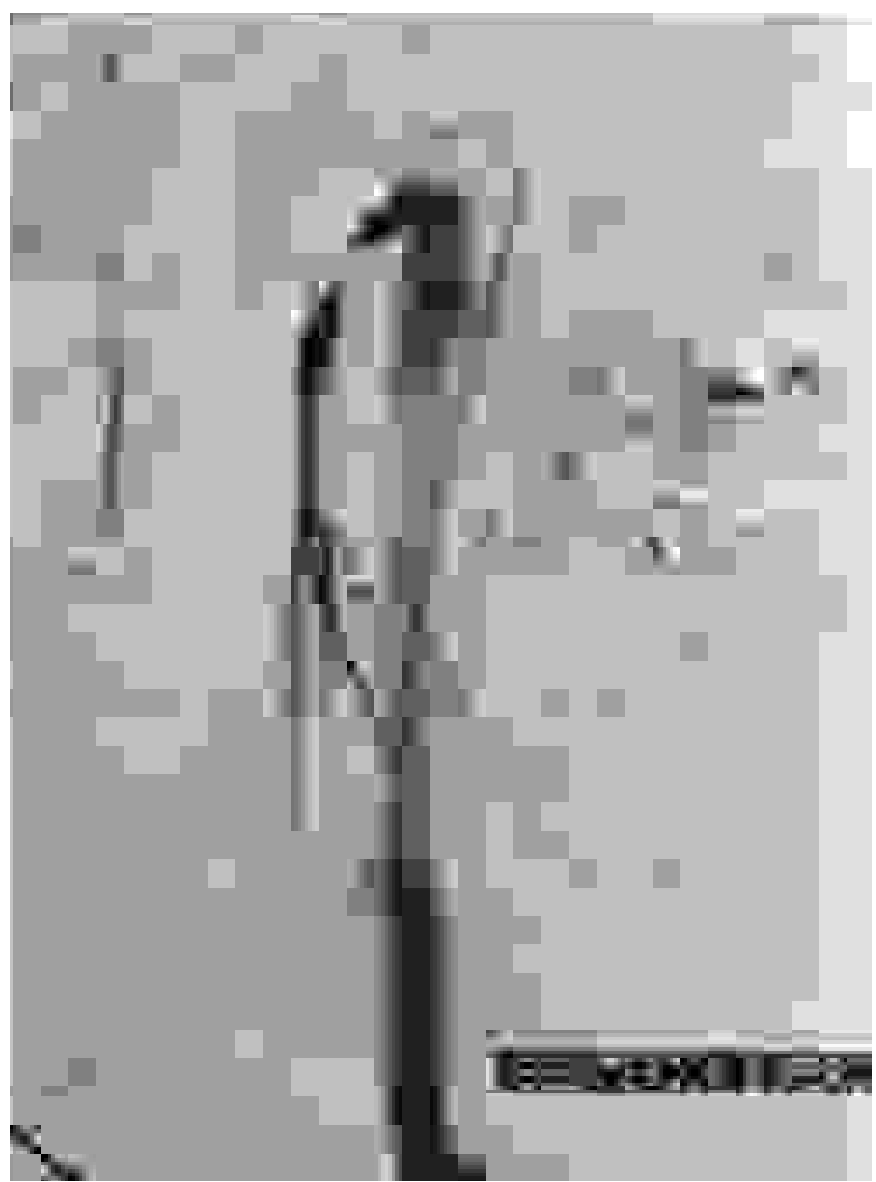

Fig. 1. Arteriografía selectiva de la arteria mesentérica superior lateral. Se observa una estenosis crítica (más del $90 \%$ de su luz) a $1 \mathrm{~cm}$ del ostium de salida.

\section{DISCUSIÓN}

La isquemia crónica intestinal es una enfermedad de escasa frecuencia debido a la gran cantidad de arterias colaterales y vasos vicariantes que existen para irrigar el intestino. La incidencia anual de la IMC en la población general se estima enl/100.000 (5). La causa más frecuente es la arterioesclerosis, siendo otras causas menos habituales la arteritis, la disección de la arteria, la displasia fibromuscular y la neurofibromatosis. Los pacientes con IMC pueden sufrir dolor postprandial, náuseas, vómitos y diarreas, síntomas de malabsorción, "terror a la comida" y progresivamente pérdida de peso por disminución de la ingesta (2).

La utilidad de la ultrasonografía doppler en la valoración de los flujos en la arteria mesentérica superior es conocida desde hace más de una década (6). No obstante, no es hasta principios de los años 90 cuando se comprueban sus aplicaciones clínicas en el diagnóstico de la isquemia mesentérica $(7,8)$. Se han determinado las velocidades normales de la AMS en sujetos sanos en condiciones de ayuno y parece claro que velocidades pico sistólicas superiores a $275 \mathrm{~cm} / \mathrm{seg}$ (7) resultan un índice claro de estenosis. En el caso que presentamos, no logramos medir la velocidad pico sistólica por dificultades técnicas, aunque la velocidad telediastólica superó los $300 \mathrm{~cm} / \mathrm{seg}$, con lo que se cumple sobradamente el criterio de estenosis. Otro punto es la utilidad que ha tenido en el caso que nos interesa el modo "angio" (o "power") en la localización del punto de estenosis ya 


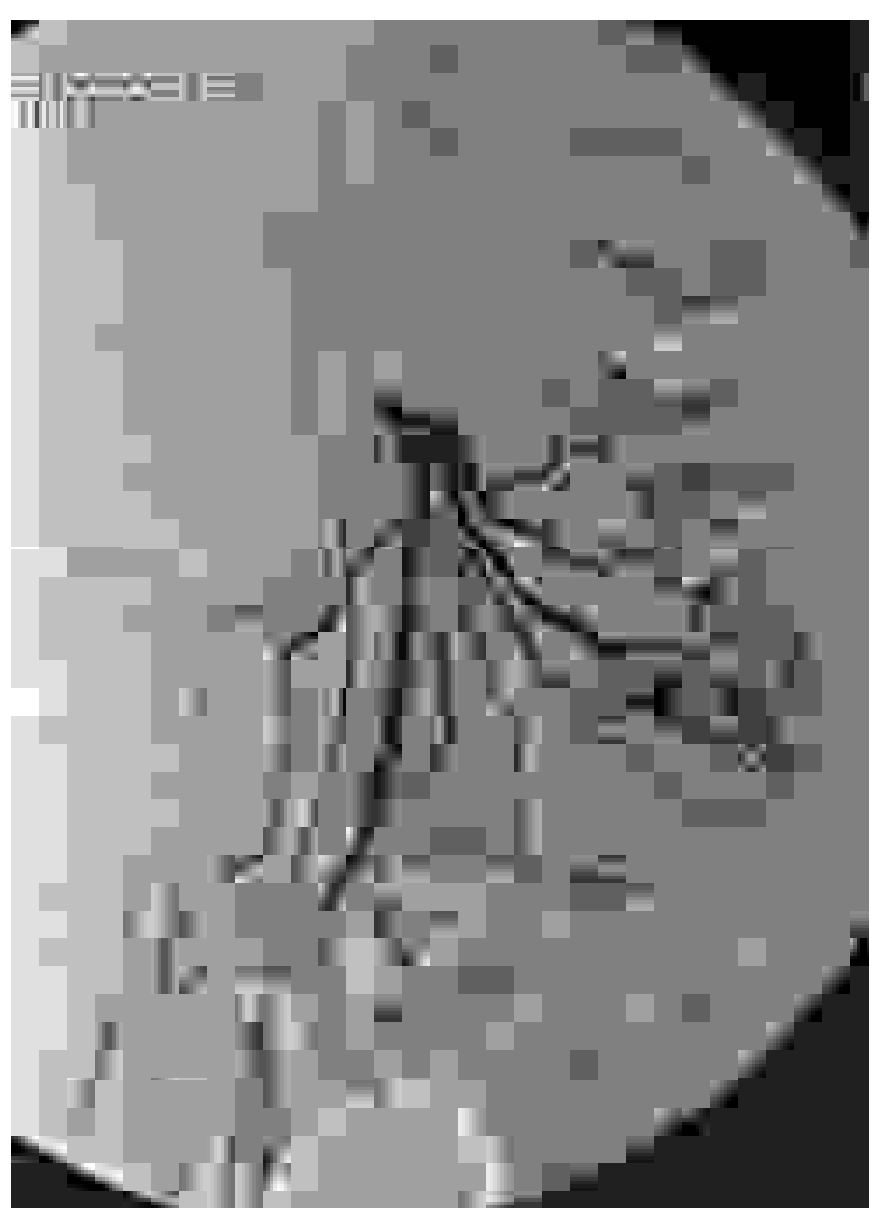

Fig. 2. Resultado post-dilatación. Se representa todas las ramas arteriales dependientes de la arteria mesentérica superior.

que al utilizar un sector convexo de color que impide corregir el ángulo de incidencia es de gran interés el disponer de una técnica independiente de ángulo que permita un correcto relleno de color del vaso sometido a estudio.

\section{Bibliografía}

1. Fisher D, Fry W. Colateral mesenteric circulation. Surg Gynecol Obstet. 1987; 164: 387-92.

2. Baxter BY, Pearce WH. Diagnosis and surgical management of chronic mesenteric ischemia. En: Strandness DE, van Breda A. Vascular diseases: Surgical and Interventional Therapy. Churchill Livengstone, New york, 1994; 795-802.

3. Matsumoto AH. Angiographiy and endovascular interventions for mesenteric ischemia. J Vasc Intervent Radiol 1996; 7 (suppl): 315-21.

4. Brandt L, Smithline A. Ischemic lesions of the bowel. En: Sleisenger M, Feldman M, Scharschmidt BF. Gastrointestinal and liver disease. Pathology/diagnosis/treatment. Saunders: Philadelphia, 1998; 2009-24.

5. Taylor LM, Moneta GM. Intestinal Ichemia. Ann Vasc Siurg 1991; 5: 403-6.

6. Jäger K, Bollinger A, Valli C, Ammann R. Measurement of mesenteric blood flow by Duplex scanning. J Vasc Surg 1986; 3: 462-9.

7. Moneta GL, Lee RW. Diagnosis of intestinal ischemia. En: Rutherford RB (ed) Vascular Surgery, $4^{\circ}$ ed. WB Saunders, Philadelphia, 1995; 1267-78.

8. Benenati JF. Intravascular untrasound: The role in diagnostic and therapeutic procedures. Radiol clin North Am 1995; 33: 31-48.

9. Taylor LM, Porter JM. Treatment of chronic visceral ischemia. En: Rutherford RD (ed). Vascular Surgery $4^{\circ}$ ed. WB Saunders, Philadelphia 1995; 1301-11.
La revascularización quirúrgica y la resección del segmento intestinal infartado han sido, hasta hace poco, el tratamiento de elección tanto en la isquemia aguda como en la crónica (3). El tratamiento estándar quirúrgico se ha llevado a cabo por revascularización de 1 ó 2 arterias mesentéricas en los pacientes sintomáticos, consiguiendo una permeabilidad primaria que varía, según las series, entre $80-85 \%$ durante un seguimiento entre 24 y 69 meses (9).

En 1980 se describió la primera angioplastia percutánea para el tratamiento de la ICM (10). Los resultados obtenidos en las diferentes series publicadas dan unas cifras medias de éxito de la técnica del $87 \%$ con unos resultados clínicos inmediatos buenos en un $85 \%$. La permeabilidad media primaria y secundaria es de 63 y $75 \%$ respectivamente (11). En cuanto a la localización de la lesión éstas se pueden dividir en ostiales y no ostiales. En las series publicadas el éxito obtenido es del $95 \%$ para las no ostiales y del 78\% para las ostiales (12-14). El uso de stents intravasculares en estos pacientes se ha limitado a los casos en los que la angioplastia percutánea ha fallado y nunca como primera opción, aunque las series publicadas son escasas (15). Esto supone un paso más en el tratamiento percutáneo y una segunda opción poco invasiva a tener en cuenta.

En cuanto a las complicaciones de la angioplastia están descritas en un 7\% de los casos, casi todas relacionadas con el sitio de punción en pacientes con abordaje axilar (hematomas, daño en el plexo braquial etc.) (11). Nosotros preferimos, si es posible un abordaje femoral que minimiza este tipo de complicaciones. En el tratamiento quirúrgico la morbilidad se ha descrito en $22-26 \%$ de los casos con una mortalidad de $16 \%$ (12), ambas cifras ostensiblemente más altas que las descritas en el tratamiento percutáneo (l 1).

Así pues, creemos que en los casos de isquemia crónica intestinal en los que se pueda demostrar una estenosis de la arteria mesentérica, la terapéutica percutánea ofrece menos complicaciones que la cirugía. Precisa únicamente anestesia local y el paciente puede ser dado de alta 24 horas después del procedimiento, ventajas todas ellas, que disminuyen el tiempo de estancia hospitalaria aumentando la comodidad del paciente.

10. Furrer J, Gruntzig A, Kugelmeier J, Goebel N. Treatment of abdominal angina with percutaneous dilatation of an arteria mesenterica superior stenosis. Cardiovasc Intervent Radiol 1980; 3: 43-4.

11. Nyman U, Ivancev K, Lindh M, Uher P. Endovascular treatment of chronic mesenteric ischemia: repost of five cases. Cardiovasc Intervent Radiol 1998; 21: 305-13.

12. Rose SC, Quigley TM, Raker EJ. Revascularization for chronic mesenteric ischemia: Comparison of operative by pass grafting and percutaneous transluminal angioplasty J Vasc Intervent Radiol 1995; 6: 339--49.

13. Hallisey MJ, Deschaine J, Illecas FF, Sussman SK, Vine HS, Ohki SK, et al. Angioplasty for the treatment of visceral ischemia. J Vasc Intervent Radiol 1995; 6: 785-91.

14. Allen RC, Martin GH, Rees CR, Rivera FJ, Talkington CM, Garrett $\mathrm{WV}$, et al. Mesenteric angioplasty in the treatment of chronic intestinal ischemia. J Vasc Surg 1996; 24; 415-23.

15. Liermann D, Strecker EP. Tantalum stents in the treatment of stenotic and occlusive diseases of abdominal vessels. In: Lermann DD (ed) Stents: State of the Art and Future Developments. Polyscience Publications, Morin heights, in conjunction with Boston Scientific, Watertown, Mass, 1995; 127-34. 